\title{
Methodische Untersuchungen zur Bestimmung der Aldolase-Aktivität im Serum
}

\author{
Von H. H. BERLET \\ Aus dem Klinisch-Chemischen Labor der Neurologischen Universitäts-Klinik Göttingen \\ (Direktor: Prof. Dr. H.J. Bauer)
}

(Eingegangen am 29. Juni 1967)

\begin{abstract}
Der Einfluß von Pyruvat auf die Ergebnisse der Bestimmung der Serum-Aldolaseaktivität in einem zusammengesetzten optischen Test nach RACKER wurde vergleichend mit den Ergebnissen eines Farbtestes untersucht. Es wurde gezeigt, daß Pyruvat im Testansatz sowohl aus dem inkubierten Serum als auch aus der Substratlösung für die Aldolasebestimmung stammen kann. Die empfohlene Vorinkubationsdauer des Reaktionsgemisches war nicht ausreichend, die Beimischungen von Pyruvat innerhalb dieser Zeit vollständig zu reduzieren, so daß im optischen Test fälschlich erhöhte Enzymaktivitäten gefunden wurden. Zur Ausschaltung der Störwirkung von Pyruvat wurde dem Testansatz Lactatdehydrogenase zugesetzt. Bei einer Vorinkubationsdauer von $5 \mathrm{Min}$. wurden mit dieser Modifikation Werte gemessen, die der tatsächlichen Aldolaseaktivität im Serum entsprachen.
\end{abstract}

The effect of pyruvate on the measurement of serum aldolase activity was studied in the coupled enzymic-optical method of RACKER, and in a colorimetric method. It was shown that pyruvate in the test mixture can arise from the incubated serum, and from the substrate solution for the aldolase determination. The recommended preincubation period for the reaction mixture was not long enough to completely remove the accompanying pyruvate, so that erroneously high enzyme activities were found by the enzyme-optical test. To prevent interference by pyruvate, lactate dehydrogenase was added to the test sample, followed by preincubation for 5 minutes. With this modification, the measured values represented the true aldolase activity of the serum.

Die Aktivitätsbestimmung der Serumaldolase findet in der Differentialdiagnose von Erkrankungen der Leber und des Muskelsystems Verwendung. Das methodische Prinzip verschiedener Verfahren zur Bestimmung der Aldolaseaktivität $t^{1}$ ) bildet die Messung der in Gegenwart des Enzyms entstehenden Triosephosphate (1-3). Bei der kolorimetrischen Methode (4) werden nach alkalischer Hydrolyse der Triosephosphate Dihydroxyaceton und Glycerinaldehyd mit 2,4-Dinitrophenylhydrazin in ein Hydrazon bzw. Osazon (5) überführt. Im optischen Test nach Warburg wird Dihydroxyacetonphosphat in Gegenwart von Glycerin-1-phosphat-Dehydrogenase und NADH zu Glycerin-1-phosphat reduziert, wobei zugesetzte Triosephosphatisomerase das bei der Fructosephosphatspaltung zu gleichen Teilen entstehende Glycerinaldehyd-3-phosphat zu Dihydroxyacetonphosphat umwandelt (6). Die mit dem Verbrauch von $\mathrm{NADH}$ verbundene Extinktionsabnahme dient als Meßgröße. Eine weitere Möglichkeit besteht darin, Glycerinaldehyd-3-phosphat durch Glycerinaldehyd-3-phosphat-Dehydrogenase in Gegenwart von Arsenat und NAD zu 3-Phosphoglycerat zu oxydieren $(7,8)$. In diesem Fall dient die Zunahme der Extinktion durch Reduktion von NAD als Meßgröße.

Infolge der unterschiedlichen Reaktionsbedingungen der verschiedenen Testansätze sind die damit gefundenen Aktivitäten und Normalbereiche nicht direkt vergleichbar. Eine Ausnahme müßten der Farbtest und der spektrophotometrische Test in der von BRUNS angegebenen

\footnotetext{
1) Der Trivialname Aldolase wird hier gebraucht für das Enzym Fructose-1,6-diphosphat D-Glycerinaldehyd-3-phosphat-Lyase (EC 4.1.2), L-Lactat-Dehydrogenase für L-Lactat: NAD Oxydoreduktase (EC 1.1.1.27); Glycerin-1-phosphat-Dehydrogenase für L-Glycerin-3-phosphat: NAD Oxydoreduktase (EC 1.1.1.8), Glycerinaldehyd-3-phosphat-Dehydrogenase für D-Glycerinaldehyd-3-phosphat: NAD Oxydoreduktase (EC 1.2.1.12); Triosephosphatisomerase für D-Glycerinaldehyd-3-phosphat-Ketol Isomerase (EC 5.3.1.1) Alkoholdehydrogenase für Alkohol: NAD Oxydoreduktase (EC 1.1.1.1).
}

Zusammensetzung $(9,10)$ bilden, da die Reaktionsbedingungen in diesem Fall übereinstimmen.

Ein experimenteller Vergleich der mit beiden Methoden gemessenen Aldolaseaktivitäten im Serum ergab jedoch stark voneinander abweichende Werte, deren Differenz bei Seren mit normaler Aktivität der Aldolase besonders deutlich war. Dabei lagen die im optischen Test gemessenen Aktivitäten höher als die des Farbtestes. Warburg und Christian (8) haben auf die Bedeutung der Brenztraubensäure als Ursache von Fehlmessungen der Aldolaseaktivität im optischen Test hingewiesen. Im folgenden wurde deswegen untersucht, ob die unterschiedlichen Ergebnisse der beiden Bestimmungsmethoden der Serum-Aldolaseaktivität auf die Gegenwart von Brenztraubensäure zurückzuführen sind. Als Resultat dieser Untersuchungen wurde der optische Test so modifiziert, daß die damit gewonnenen Werte der wahren Aldolaseaktivität im Serum entsprechen.

\section{Methodik}

Für die Bestimmungen der Aldolaseaktivität im hämolysefreien Serum wurde venöses Nüchternblut ohne Zusätze verwendet. Die Seren wurden bis zu ihrex Aufarbeitung, die in jedem Fall am Tag der Blutentnahme durchgeführt wurde, im Kühlschrank bei $4^{\circ}$ aufbewahrt.

\section{Bestimmung der Aldolaseaktivität}

\section{Farbtest}

Der Farbtest wurde nach Sibley und Lehninger (4) in der von BRUNS angegebenen Modifikation $(9,10)$ ausgeführt (Testkombination der Fa. Boehringer \& Soehne, Mannheim). Der Testansatz enthielt in $2,0 \mathrm{~m} / 0,004 \mathrm{M}$ Collidinpuffer ( $\mathrm{pH} 7,4) 0,025 \mathrm{mMol}$ Fructose-1,6-diphosphat, $0,14 \mathrm{mMol}$ Hydrazinsulfat und $0,4 \mu \mathrm{Mol}$ Jodacetat. Die Reaktion wurde mit $1,0 \mathrm{~m} /$ Serum gestartet; nach $60 \mathrm{Min}$. Inkubation bei $37^{\circ}$ wurde die Reaktion durch Zugabe von Trichloressigsäure gestoppt, der Überstand durch Zusatz von $0,75 \mathrm{~N} \mathrm{NaOH}$ im Uberschuß alkalisch gemacht ( $\mathrm{pH} 12-13)$, nach 10 Min. Hydrolyse mit einer Lösung von $0,1 \%$ 2,4-Dinitrophenylhydrazin in $2 \mathrm{~N} \mathrm{HCl}$ versetzt, erneut $10 \mathrm{Min}$. bei $37^{\circ}$ inkubiert und danach mit $0,75 \mathrm{~N} \mathrm{NaOH}$ im Uberschuß alkalisch gemacht. Die so entwickelte Farbe wurde bei $530-550 \mathrm{~nm}$ (Eppendorf Filter $546 \mathrm{~nm}$ ) gemessen (s. a. Testvorschrift). 


\section{Optischer Test (6)}

$\mathrm{Zu} 2,5 \mathrm{~m} / 0,056 \mathrm{M}$ Collidinpuffer ( $\mathrm{pH} 7,4)$, der 7,5 $\mu$ Mol Fructose1,6-diphosphat und $0,75 \mu \mathrm{Mol}$ Jodacetat enthielt, wurden $0,05 \mathrm{ml}$ einer $0,020 \mathrm{M} \mathrm{NADH}$-Lösung ( $1 \mu \mathrm{Mol} \mathrm{NADH}$ ) sowie $0,01 \mathrm{ml}$ eines Glycerin-1-phosphat-Dehydrogenase/Triosephosphatisomerase-Gemisches, $2 \mathrm{mg} / \mathrm{m} /$, hinzugefügt (Testkombination der $\mathrm{Fa}$. Boehringer \& Soehne, Mannheim). Die Reaktion wurde mit $0,2 \mathrm{~m} /$ Serum gestartet. Die Aktivität wurde aus der Extinktionsabnahme bei $366 \mathrm{~nm}$ und $37^{\circ}$ zwischen der 1. und 21. Min. nach Serumzusatz berechnet.

\section{Bestimmung von Pyruvat}

Pyruvat wurde mit Hilfe von Lactatdehydrogenase in Gegenwart von $\mathrm{NADH}$ in $0,1 \mathrm{M}$ Phosphatpuffer ( $\mathrm{pH} 7,5$ ) bestimmt (11).

Darstellung und Dünnschichtchromatographie der 2,4-Dinitrophenylhydrazone von Ketosäuren

Zum Nachweis von Ketosäuren, speziell von solchen, die durch Lactatdehydrogenase rasch hydriert werden (12), wurden Oxalessigsäure, Brenztraubensäure, Glyoxylsäure, $\beta$-Hydroxypyruvat und $\alpha$-Ketobuttersäure für Vergleichszwecke als 2,4-Dinitrophenylhydrazone nach Standardmethoden (13) dargestellt. Ketosäuren in Lösungen unbekannter Zusammensetzung wurden ebenfalls in Hydrazone überführt und mit Essigsäureäthylester extrahiert (14). Standardsubstanzen und Extrakte wurden zweidimensional auf Kieselgel (DC-Fertigplatten, E. Merck AG, Darmstadt) in n-Butanol/Äthanol/0,5N Ammoniak 70:10:20 (v/v) und Benzol/ Tetrahydrofuran/Eisessig 57:35:8 (v/v) aufgetrennt (15).

Enzyme, NADH, Fructose-1,6-diphosphat und Brenztraubensäure stammten von der Fa. Boehringer \& Soehne, Mannheim, Oxalessigsäure von Serva, Heidelberg, $\alpha$-Ketobuttersäure von Sigma Chemical Company, St. Louis, USA, Glyoxylsäure von Fluka, Buchs, Schweiz, und $\beta$-Hydroxybrenztraubensäure von Nutritional Biochemicals, Cleveland, Ohio, USA.

Für die kolorimetrischen und optischen Messungen stand ein Photometer Eppendorf der Fa. Netheler und Hinz zur Verfügung. Die statistische Signifikanz der Unterschiede von Mittelwerten wurde mithilfe des $t$-Testes (Student-Test) berechnet.

\section{Ergebnisse}

\section{Vergleich zivischen Farbtest und UV-Test}

Tabelle 1 zeigt eine Gegenüberstellung von Aldolaseaktivitäten in 9 verschiedenen Seren, die einmal mit Hilfe des Farbtestes und zum anderen im UV-Test gemessen wurden. Der UV-Test ergab für alle Seren

Tab. 1

Vergleichende Bestimmung der Aldolaseaktivität im Serum nach der Methode des Farb testes und des UV-Testes

\begin{tabular}{|c|c|c|c|}
\hline . & $\begin{array}{l}\text { Farbtest } \\
\mathrm{mU} / \mathrm{m} l\end{array}$ & $\mathrm{D}_{\mathrm{t}} \underbrace{\mathrm{UV}}_{\mathrm{ml}}$ & $\begin{array}{l}\text { Test } \\
/ \mathrm{ml}_{\mathrm{t}} 11-21 \mathrm{Min} \cdot 2\end{array}$ \\
\hline $\begin{array}{c}\text { Aldolase, } \\
\text { krist. (5 mg/ml) } \\
1: 40000 \\
1: 20000\end{array}$ & $\begin{array}{l}1,58 \\
3,72\end{array}$ & $\begin{array}{l}1,87 \\
4,11\end{array}$ & $\begin{array}{l}1,85 \\
4,01\end{array}$ \\
\hline \multicolumn{4}{|l|}{ Serum* ${ }^{*}$} \\
\hline 1 & 1,66 & 3,53 & 2,26 \\
\hline 2 & 1,77 & 3,42 & 2,15 \\
\hline 3 & 1,42 & 3,36 & 2,64 \\
\hline 4 & 1,55 & 3,61 & 1,98 \\
\hline 5 & 1,30 & 3,44 & 1,81 \\
\hline 6 & 1,20 & 2,75 & 1,38 \\
\hline 7 & 1,32 & 3,01 & 1,72 \\
\hline 8 & 1,13 & 3,44 & 2,28 \\
\hline$M \pm s$ & $1,42 \pm 0,22^{* *}$ & $3,32 \pm 0,29$ & $2,03 \pm 0,39 * *$ \\
\hline
\end{tabular}

* Mittelwerte aus Doppelbestimmungen in 8 verschiedenen Seren. ** $\mathrm{p}<0,05$ für den Unterschied der Spalten 1 und 3 gegen Spalte 2. Der Unterschied zwischen Spalte 1 und 3 ist nicht signifikant. wesentlich höhere Werte als der Farbtest. In manchen Fällen (Serum 5 und 8) beträgt die Differenz fast das dreifache des Farbtestes, in allen übrigen Beispielen mindestens das zweifache. Eine bessere Angleichung an die Werte des Farbtestes erhält man, wenn man die Extinktionsabnahme zwischen der 11. und 21. Min. für die Berechnung der Enzymaktivität berücksichtigt (Tab. 1, 3. Spalte). Dagegen zeigen die Ergebnisse mit einem reinen, stark verdünnten Aldolasepräparat, daß die mit beiden Methoden gefundenen Werte gut übereinstimmen.

Der Verlauf der Extinktionsabnahme im UV-Test nach Zusatz von Serum bzw. reiner Aldolase ist in Abbildung 1 graphisch dargestellt. Der Extinktionsabfall

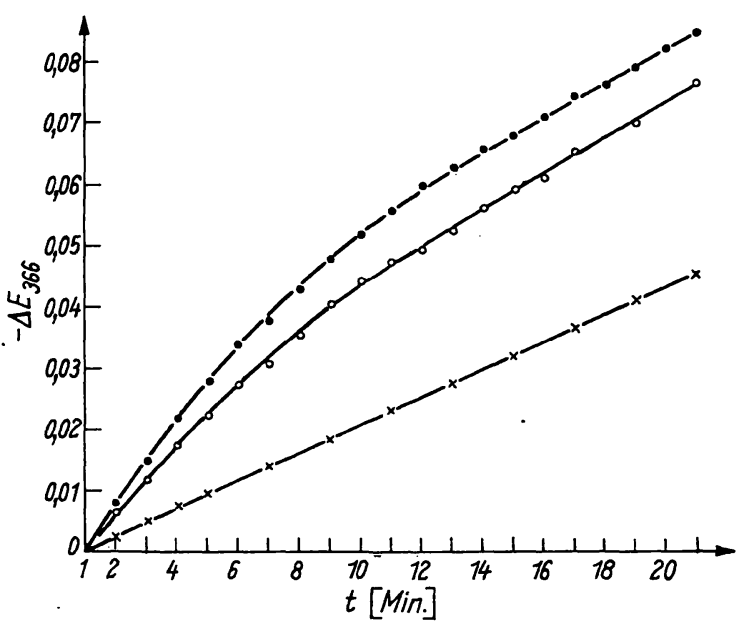

Abb. 1

Verlauf des Extinktionsabfalles im Testansatz für die Bestimmung der Aldolaseaktivität

Das Reaktionsgemisch enthielt 7,5 $\mu \mathrm{Mol}$ Fructose-1,6-diphosphat, $1,0 \mu \mathrm{Mol}$ NADH, $0,75 \mu \mathrm{Mol}$ Jodacetat und $0,01 \mathrm{ml}$ Triosephosphatisomerase/Glycerin- 1 -phosphat-Dehydrogenase, $2 \mathrm{mg} / \mathrm{ml}$, in einem Gesamtvolumen von $2,56 \mathrm{ml} 0,056 \mathrm{M}$ Collidinpuffer, $\mathrm{pH} 7,4$; die $\mathrm{Re}$ aktion wurde mit $0,2 \mathrm{ml}$ Serum bzw. einer verdünnten Aldolasesuspension gestartet

- Nativserum; 0 Serum, dialysiert; $\quad \times-\times$ Aldolase, $1: 20000$ mit 0,9 proz. NaCl-Lösung verdünnt

wurde im Fall des Serums erst nach 12 bis 14 Min. linear. Diese Beobachtung konnte mit zahlreichen Seren regelmäßig reproduziert werden. Durch diesen Verlauf wird innerhalb der Meßperiode eine höhere Extinktionsdifferenz vorgetäuscht als der wahren Aldolaseaktivität im Serum entspricht. Mit einer reinen Aldolaseverdünnung wat der Verlauf der Reaktion von Beginn an linear.

Aus der Abbildung ist ebenfalls der Reaktionsverlauf nach Zusatz eines Serums ersichtlich, das 12 Stdn. bei $3^{\circ}$ gegen $3000 \mathrm{ml} 0,02 \mathrm{M}$ Phosphatpuffer $(\mathrm{pH} \mathrm{7,3)}$ und $0,15 \mathrm{M} \mathrm{NaCl}$ dialysiert wurde (Visking Dialysierschlauch, Serva, Heidelberg). Der Großteil des unspezifischen NADH-Verbrauches war auch nach der Dialyse noch vorhanden. Daraus wurde gefolgert, daß nicht nur Serumbestandteile, sondern auch Reagenzien des Testansatzes zur Vorreaktion beitragen. Diese Annahme bestätigte der in Abbildung 2 dargestellte Versuch, dem vor Zugabe von Serum $0,01 \mathrm{ml}$ einer Lactatdehydrogenase $(2 \mathrm{mg} / \mathrm{ml})$ zugesetzt wurde. Eine Gegenüber- 


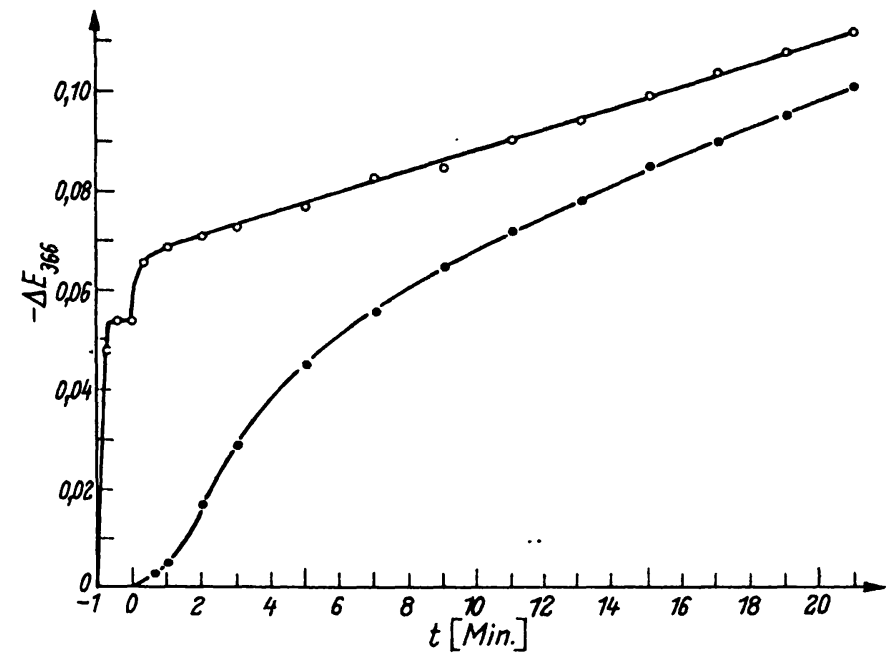

Abb. 2

Bestimmung der Aldolaseaktivität im Serum. Zusammensetzung des Testgemisches wie in Abbildung 1

- - Normaler Ansatz mit Serum; $\multimap$ Zusatz von $0,01 \mathrm{ml}$ Lactatdehydrogenase, $2 \mathrm{mg} / \mathrm{ml}$, $1 \mathrm{Min}$. vor Zugabe des gleichen Serums wie

stellung des gleichen Serums ohne Zusatz von Lactatdehydrogenase zeigt, daß in diesem Fall Linearität als Ausdruck einer Reaktion erster Ordnung erșt nach mehr als $10 \mathrm{Min}$. erreicht wurde.

Aus den Ergebnissen dieser Experimente wurde deutlich, daß die Ursache der zusätzlichen Extinktionsabnahme vor allem in der Substratlösung des optischen Aldolasetestes zu suchen ist. Die Substratlösung wurde daraufhin auf ihren Gehalt an Ketosäure untersucht.

\section{Isolierung und Dünnschichtcbromatograpbie von Brenqtrauben-} säure

Die Ketosäuren einer angesetzten Substratlösung $(20 \mathrm{ml})$ wurden als Dinitrophenylhydrazone extrahiert und dünnschichtchromatographisch aufgetrennt. Ini Chromatogramm des Extraktes kam ein deutlicher Fleck zur Darstellung, dessen $R_{\mathrm{F}}$-Werte in 2 Taufmitteln $(0,46 /$ $0,38)$ mit denen der Brenztraubensäure $(0,45 / 0,38)$ übereinstimmten.

Der Gehalt an Brenztraubensäure wurde quantitativ in 4 Substratlösungen verschiedenen Alters (8-189 Tage bei $4^{\circ}$ gelagert) bestimmt. Die Konzentration von $0,212-0,262 \mathrm{mg} / 100 \mathrm{ml}$ war in allen Lösungen annähernd gleich, ohne daß sich ein Zusammenhang zwischen Pyruvatgehalt und Alter der Lösung erkennen ließ.

Einfluis von 2,4,6-Trimetbylpyridin (2,4,6-Collidin) auf die Lactatdebydrogenase-Reaktion

Die bisherigen Untersuchungen haben gezeigt, welche Bedeutung die Brenztraubensäure als Ursache für Fehlbestimmungen der Aldolaseaktivität im UV-Test hat. Als Kontrolle erschien es notwendig, den Einfluß anderer Faktoren zu prüfen. Der Einfluß des Collidinpuffers auf die Anfangsgeschwindigkeit der Pyruvatreduktion durch Lactatdehydrogenase wurde im Vergleich mit einem Phosphatpuffer untersucht (Abb. 3). Die Ab-

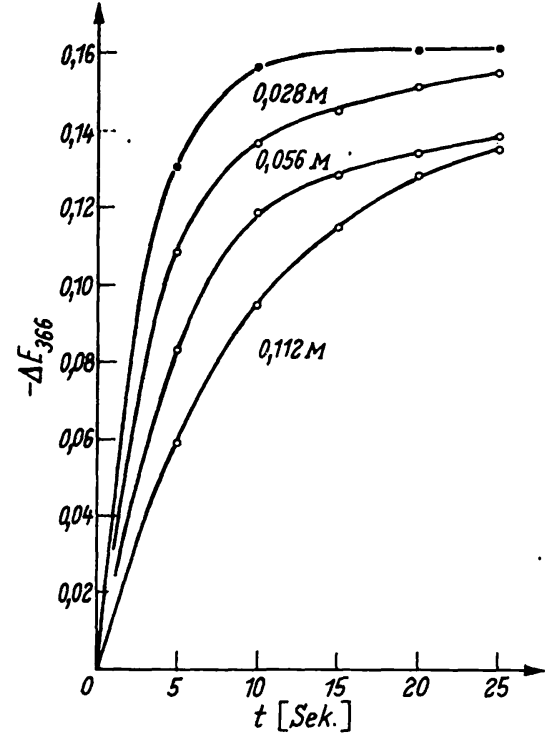

Abb. 3

Anfangsgeschwindigkeit der Pyruvathydrierung durch Kaninchenmuskel-Lactatdehydrogenase bei $25^{\circ}$ in $0,1 \mathrm{M}$ Phosphatpuffer, $\mathrm{pH} 7,5(\bullet-\bullet)$ und in Gegenwart verschiedener Konzentrationen von 2,4,6-Trimethylpyridin (०-o), eingestellt auf $\mathrm{pH} 7,5$

Das Reaktionsgemisch von $3,0 \mathrm{ml}$ enthielt $0,157 \mu \mathrm{Mol}$ Pyruvat und $0,6 \mu \mathrm{Mol} N \mathrm{NAH}$; die Reaktion wurde mit $0,01 \mathrm{ml}$ Lactatdehydrogenase $(2 \mathrm{mg} / \mathrm{ml})$ gestartet

bildung zeigt, daß Collidinkonzentrationen zwischen 0,028 und 0,112 $\mathrm{M}$ die Pyruvatreduktion deutlich hemmen. Diese Hemmung wirkt sich jedoch auf den gesamten zeitlichen Ablauf der Pyruvatreduktion im Rahmen der Aldolasemessung nicht weiter verzögernd aus. Diese Tatsache ging aus Experimenten hervor, bei denen gleiche Seren einmal in dem üblichen Collidinpuffer und daneben in einem Tris-HCl-Puffer $(0,05 \mathrm{M}, \mathrm{pH} 7,4)$ inkubiert wurden. In diesen Experimenten wurden keine Unterschiede für die Zeitdauer bis zur vollständigen Hydrierung von Pyruvat gefunden.

\section{Messung der Serum-Aldolaseaktivität unter Zısatz, von Lactatdehyidrogenase}

Die in Abbildung 2 gezeigte Wirkung eines Zusatzes von Lactatdehydrogenase auf den Verlauf der Pyruvatreduktion wurde im folgenden dazu benutzt, in einer Serie von vergleichenden Bestimmungen den störenden Einfluß von Brenztraubensäure auszuschalten. Das Testgemisch in der üblichen Zusammensetzung wurde mit $0,01 \mathrm{ml}$ Lactatdehydrogenase-Suspension $(2 \mathrm{mg} / \mathrm{ml}) 5$ Min. vorinkubiert. Danach wurde die Reaktion mit $0,2 \mathrm{ml}$ Serum gestartet und der Extinktionsabfall zwischen der 1. und 21. Min. nach Serumzugabe gemessen. Die Aktivität der gleichen Seren wurde daneben im Farbtest und im UV-Test ohne Zusatz von Lactatdehydrogenase bestimmt (Tab. 2). Die Meßwerte des Farbtestes und des modifizierten UV-Testes zeigten eine gute, statistisch gesicherte Übereinstimmung. Dagegen sind die Werte des üblichen UV-Testes wieder im Mittel um das Doppelte höher. Bei echten Erhöhungen der Aldolaseaktivität (Serum 5) scheinen sich die verschiedenen Werte anzunähern. 
Tab. 2

Vergleichende Bestimmung der Aldolaseaktivität im Serum nach der Metlode des Farbtestes und des UV-Testes, ohne und mit Zusatz von Lactatdehydrogenase (LDH)

\begin{tabular}{|c|c|c|c|}
\hline Serum** & $\begin{array}{l}\text { Farbtest } \\
\mathrm{mU} / \mathrm{ml}\end{array}$ & $\begin{array}{c}\text { UV-Test mit } \\
\text { Zusatz von LDH } \\
\mathrm{mU} / \mathrm{ml} l^{*}\end{array}$ & $\begin{array}{c}\text { UV-Test ohne } \\
\text { Zusatz von LDH } \\
\text { mU/m } / l^{*}\end{array}$ \\
\hline $\begin{array}{r}1 \\
2 \\
3 \\
4 \\
5 \\
6 \\
7 \\
8 \\
9 \\
10 \\
11 \\
12\end{array}$ & $\begin{array}{l}2,11 \\
2,20 \\
1,57 \\
1,62 \\
4,95 \\
0,42 \\
1,63 \\
1,23 \\
0,86 \\
0,73 \\
0,99 \\
2,60\end{array}$ & $\begin{array}{l}2,10 \\
2,00 \\
1,85 \\
1,70 \\
4,00 \\
0,47 \\
1,70 \\
1,24 \\
0,90 \\
0,81 \\
1,11 \\
2,70\end{array}$ & $\begin{array}{l}4,10 \\
4,22 \\
4,26 \\
3,78 \\
4,12 \\
2,15 \\
3,50 \\
3,18 \\
2,49 \\
2,36 \\
2,83 \\
4,81\end{array}$ \\
\hline$M \pm s:$ & $1,74 \pm 1,20$ & $1,72 \pm 1,04$ & $\begin{array}{c}3,48 \pm 0,87 \\
p<0,01\end{array}$ \\
\hline
\end{tabular}

- Die Aktivität wurde aus der Extinktionsdifferenz zwischen der 1. und 21. Min. nach Zusatz des Serums berechnet. Test einfach bestimmt.

Der Unterschied der Mittelwerte in Spalte 1 und 2 ist nicht signifikant.

\section{Diskussion}

Es wurde gezeigt, daß die unterschiedlichen Meßergebnisse eines Farbtestes und eines UV-Testes für die Bestimmung der Aldolaseaktivität im Serum auf fälschlich erhöhte Meßwerte im UV-Test zurückzuführen sind. Als Ursache hierfür wurde die nicht zu umgehende Beimischung von Brenztraubensäure zum Ansatz gefunden, die in den Testvorschriften nicht genügend Beachtung findet, obwohl auf die Bedeuturg der Brenztraubensäure in diesem Zusammenhang bereits früher hingewiesen wurde $(8,16)$.

Die Vermutung, daß andere, mit Lactatdehydrogenase langsam reagierende Ketosäuren (12) zur Dauer der Vorreaktion beitragen, konnte durch die chromatographischen Untersuchungen ausgeschlossen werden. Ebenso spielt die gefundene Hemmung der Lactatdehydrogenase durch Collidin nur eine untergeordnete Rolle. Der langsame Ablauf der Vorreaktion ist demnach allein durch die in normalen Seren nicht sehr hohe Lactatdehydrogenase-Aktivität bedingt. Zur vollständigen Hydrierung der im Testansatz vorhandenen Brenztraubensäure wä- ren deswegen wesentlich längere Vorinkubationszeiten erforderlich als die in den Arbeitsvorschriften empfohlenen. Da Pyruvatgehalt und Aktivität der Lactatdehydrogenase von Serum zu Serum sehr verschieden sein können, erscheint der Zusatz von Lactatdehydrogenase im Überschuß als die beste Möglichkeit, die Störung der Aldolasereaktion durch die Brenztraubensäure in gut reproduzierbarer Form auszuschalten. Eine vollständige Hemmung der Lactatdehydrogenase durch einen spezifischen Inhibitor wäre eine andere Möglichkeit. Vorläufige Experimente mit Laurylsulfat, das als wirksamer Lactatdehydrogenase-Inhibitor verwendet wird (17), schlugen fehl, da der zusammengesetzte optische Aldolasetest ebenfalls gehemmt wurde. Die selektive Hitzeinaktivierung der Lactatdehydrogenase ist ebenfalls nicht möglich, da sich Lactatdehydrogenase und Aldolase in ihrer Thermostabilität sehr ähnlich verhalten $(1,11)$. Die Beobachtung von erhöhten Aldolasewerten, die sich weder klinisch noch durch andere Laborfunde erklären lassen, hat zu der Empfehlung gefühtt, die obere Grenze des Normalbereiches der im UV-Test gemessenen Enzymaktivitäten von 2,5 auf $6 \mathrm{mU} / \mathrm{m} l$ Serum zu erhöhen. Diese Maßnahme erscheint im Hinblick auf die Ergebnisse dieser Untersuchungen nicht gerechtfertigt. Sie hätte zur Folge, daß Seren mit niedrigem Pyruvatgehalt und hoher LactatdehydrogenaseAktivität, die das vorhandene Pyruvat in weniger als 5 Min. reduzieren, fälschlich als normal eingestuft würdẹn, selbst wenn die tatsächliche Aldolaseaktivität im Bereich von 2,5 bis $6 \mathrm{mU} / \mathrm{ml}$ liegt, der nach der ursprünglichen Auffassung bereits als pathologisch zu betrachten ist.

Die Hemmung der Lactatdehydrogenase durch Pyridinbasen, die in 2,4,6-Stellung substituiert sind, wurde bisher nicht beschrieben. Dagegen ist die Hemmung der Alkoholdehydrogenase durch freie Pyridinbasen und $\mathrm{N}^{\prime}$-Methylpyridinderivate bekannt (18), sowie eine Hemmung der Hefe-Alkoholdehydrogenase durch 4Methylpyridin (19).

Frau I. LAU danke ich für ihre technische Mitarbeit.

\section{Literatur}

1. Meyerhof, O. und K. Lohmann, Biochem. Z. 271, 89 (1934). 2. Meyerhof, O., L. Lohmann und P. Schuster, Biochem. Z. 286, 301 (1936). - 3. MeYerhof, O., K. LohmanN und P. Schuster, Biochem. Z. 286, 319 (1936). - 4. Sibley, J. A. und A. L. LeHNINGER, J. biol. Chemistry 177, 859 (1949). - 5. BECK, W. S., J. biol. Chemistry 212, 847 (1955). - 6. Racker, E., J. biol. Chemistry 167, 843 (1947). - 7. Warburg, O. und W. Christian, Biochem. Z.314, 149 (1943). - 8. Warburg, O. und W. Christian, Biochem. Z. 314, 399 (1943). - 9. Bruns, F., Biochem. Z. 325, 156 (1954). - 10. Bruns, F., Biochem. Z. 325, 429 (1954). - 11. Kubowitz, F. und P. Otr, Biochem. Z. 314, 94 (1943). - 12.
Meister, A., J. biol. Chemistry 184, 117 (1950). - 13. El Hawary, M. F. S. und R. H. S. Thompson, Biochem. J. 53, 340 (1953). 14. BAChelARD, H. S., Nature (London) 205, 903 (1965). - 15. Berlet, H. H., Anal. Biochem. Im Druck. - 16. Delbrück, A., E. Zebe und T. Bücher, Biochem. Z. 331, 273 (1959). - 17. Weber, W. W. und V. Zannon,, J. biol. Chemistry 241, 1345 (1966). - 18. WeBB, J. L., Enzyme and Metabolic Inhibitors, Band II S. 484. Academic Press, New York (1966). - 19. VAN EYs, J. und N. O. KapLAN, Biochim. biophysica Acta (Amsterdam) 23, 574 (1957).

Dr. H. Berlet

69 Heidelberg, Berliner Straße 5 Postfach 1368 